\title{
Language Models as Knowledge Bases: On Entity Representations, Storage Capacity, and Paraphrased Queries
}

\author{
Benjamin Heinzerling ${ }^{1,2}$ and Kentaro Inui ${ }^{2,1}$ \\ ${ }^{1}$ RIKEN AIP \& ${ }^{2}$ Tohoku University \\ benjamin.heinzerling@riken.jp | inui@tohoku.ac.jp
}

\begin{abstract}
Pretrained language models have been suggested as a possible alternative or complement to structured knowledge bases. However, this emerging LM-as-KB paradigm has so far only been considered in a very limited setting, which only allows handling $21 \mathrm{k}$ entities whose name is found in common LM vocabularies. Furthermore, a major benefit of this paradigm, i.e., querying the $\mathrm{KB}$ using natural language paraphrases, is underexplored. Here we formulate two basic requirements for treating LMs as KBs: (i) the ability to store a large number facts involving a large number of entities and (ii) the ability to query stored facts. We explore three entity representations that allow LMs to handle millions of entities and present a detailed case study on paraphrased querying of facts stored in LMs, thereby providing a proof-of-concept that language models can indeed serve as knowledge bases.
\end{abstract}

\section{Introduction}

Language models (LMs) appear to memorize world knowledge facts during training. For example, BERT (Devlin et al., 2019) correctly answers the query "Paris is the capital of [MASK]" with "France". This observation prompted Petroni et al. (2019) to ask if LMs can serve as an alternative or complement to structured knowledge bases (KBs), thereby introducing the idea of treating LMs as KBs: During training, the LM encounters world knowledge facts expressed in its training data, some of which are stored in some form in the LM's parameters. After training, some of the stored facts can be recovered from the LM's parameters by means of a suitable natural language query (Fig. 1). A LM with such a "built-in" KB is useful for knowledge-intensive tasks (Petroni et al., 2020) and question answering (Roberts et al., 2020), and could improve natural language interfaces to structured data (Hendrix et al., 1978; Herzig et al., 2020).

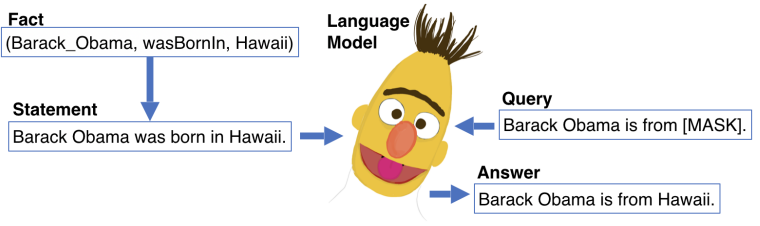

Figure 1: The LM-as-KB paradigm, introduced by Petroni et al. (2019). A LM memorizes factual statements, which can be queried in natural language.

However, this emerging LM-as-KB paradigm is faced with several foundational questions.

First question: KBs contain millions of entities, while LM vocabulary size usually does not exceed 100k entries. How can millions of entities be represented in LMs? Petroni et al. (2019) circumvent this problem by only considering $21 \mathrm{k}$ entities whose canonical name corresponds to a single token in the LM's vocabulary, e.g., entities like "France" or "Bert", but not "United Kingdom" or "Sesame Street". Hence, this approach cannot handle entities not contained in the vocabulary and a query like "Bert is a character on [MASK]" is not answerable in this simplified setting. To answer this first question, we compare three methods for scaling LM-as-KB to millions of entities:

1. Symbolic representation, i.e., extending the LM vocabulary with entries for all entities;

2. Surface form representation, i.e., each entity is represented by its subword-encoded canonical name, which is stored and queried by extending the LM with a sequence decoder; and

3. Continuous representation, i.e., each entity is represented as an embedding.

We find that, while all three entity representations allow LMs to store millions of world-knowledge facts involving a large number of entities, each representation comes with different trade-offs: Sym- 
bolic representation allows the most accurate storage, but is computationally expensive and requires entity-linked training data. Surface representation is computationally efficient and does not require entity-linked data, but is less accurate, especially for longer entity names. Continuous representation also requires entity-linked data, but is computationally more efficient than symbolic representation.

Second question: What is the capacity of LMs for storing world knowledge? Can a LM store, say, all relation triples contained in a KB like Wikidata (Vrandečić and Krötzsch, 2014)? Here we conduct experiments using synthetic data to study the scaling behaviour of current LM architectures. Varying the number of trainable model parameters and recording the number of relation triples memorized at a given accuracy level, we find that, e.g., a Transformer (Vaswani et al., 2017) with 125 million parameters (12 layers of size 768), has the capacity to memorize 1 million Wikidata relation triples with 95 percent accuracy or 5 million relation triples with 79 percent accuracy. Assuming linear scaling, this finding suggests that larger LMs with tens or hundreds of billions of parameters (Raffel et al., 2019; Brown et al., 2020) can be used to store sizable parts, if not all, of a KB like Wikidata.

Third question: How robustly is world knowledge stored in LMs? Is the LM able to recall a fact even if the query is slightly different than what was memorized during training? For example, if the LM memorized "Barack Obama was born in Hawaii" during training, can it answer queries like "Barack Obama is from [MASK]" or "Where was Barack Obama born? [MASK]"? Here we conduct experiments to measure how well the LM transfers knowledge from memorized statements to query variants, both in a zero-shot setting in which the model is not exposed to the target query variant during training, and a few shot setting, in which the model is finetuned on a small number of statements containing the target query variant. We observe zero-shot transfer in case of highly similar query variants, and see successful few-shot transfer after finetuning with 5 to 100 instances in case of less similar queries. This ability to handle soft, natural language queries, as opposed to hard, symbolic queries in a language like SQL or SPARQL, is one of the key motivations for using LMs as KBs.

Contributions. We formulate two requirements for treating LMs as KBs: (i) the ability to store a large number of facts involving a large number of entities and (ii) the ability to query stored facts. After providing background on world knowledge in LMs (§2), we make the following contributions: ${ }^{1}$

- A comparison of entity representations for scaling LM-as-KB to millions of entities (§3);

- Empirical lower bounds on LM capacity for storing world knowledge facts (§4); and

- A controlled study of knowledge transfer from stored facts to paraphrased queries $(\S 5)$.

Terminology. In this work we are interested in storing and retrieving world knowledge facts in and from a LM. World knowledge is knowledge pertaining to entities, such as Barack_Obama. A fact is a piece of world knowledge that can be expressed with a concise natural language statement, such as the English sentence Barack Obama was Born in Hawaii, or with a relation triple, such as 〈Barack_Obama, wasBornIn, Hawaii . A relation triple, or relation for short, consists of a subject entity (Barack_Obama), a predicate (wasBornln), and an object entity (Hawaii). A knowledge base is a set of relations. Knowledge bases, such as Wikidata, typically contain thousands of predicates, millions of entities, and billions of relations.

\section{World Knowledge in Language Models}

Large pretrained LMs have been the driver of recent progress in natural language processing (Peters et al., 2018; Howard and Ruder, 2018; Radford et al., 2019; Devlin et al., 2019). While the trend towards larger LMs is likely to continue (Raffel et al., 2019; Kaplan et al., 2020; Brown et al., 2020), it has limitations: (i) A model trained only on text lacks grounding in perception and experience and hence cannot learn meaning (Bender and Koller, 2020). (ii) Reporting bias leads to certain knowledge rarely or never being expressed in text. For example, a LM will easily learn to associate the phrase "Barack Obama" with the phrase "U.S. President", but might less likely learn that he is a "human being", since the latter fact is rarely stated explicitly in text. In contrast, this type of knowledge is readily available in KBs. (iii) A large number of rare entities (Hoffart et al., 2014; Derczynski et al., 2017; Ilievski et al., 2018) are, by definition, rarely mentioned, making it difficult for

\footnotetext{
${ }^{1}$ Code available at: https://github.com/bheinzerling/lm-as-kb
} 
LMs to acquire knowledge about this long tail of entities from text alone.

These limitations have motivated efforts to explicitly $^{2}$ equip LMs with world knowledge. Table 2 (Appx. A) situates these efforts on a spectrum from purely text-based LMs to representations of structured KBs. Models based on text generation (Raffel et al., 2019; Roberts et al., 2020) and retrieval (Guu et al., 2020) have proven most successful in knowledge-intensive tasks. However, we argue that models which reify entities (Logan et al., 2019), i.e., models in which entities are "first-class citizens" that can be directly predicted ${ }^{3}$, are a promising research direction, since the direct links into a $\mathrm{KB}$ can be seen as a form of grounding. This is one of our main motivations for considering symbolic and continuous entity representations.

\section{Entity Representations}

How can millions of entities be represented in a LM? To answer our first question, we compare three types of entity representations: symbolic, surface form, and continuous.

Experimental setup. We evaluate entity representations by measuring how well they allow a LM to store and retrieve world knowledge facts. For example, if the LM's training data contains the statement "Bert is a character on Sesame Street", the model should memorize this statement and recall the correct object Sesame_Street when asked with a query like "Bert is a character on [MASK]." Synthetic data. It is not a priori clear how many facts a text from the LM's training data, say, a Wikipedia article, expresses. Since we want to precisely measure how well a LM can store and retrieve facts, we create synthetic data by generating statements from $\mathrm{KB}$ relations and then train the model to memorize these statements. Using Wikidata as KB, we first define two sets of entities: A smaller set consisting of the top 1 million Wikidata entities according to node outdegree, and a larger set consisting of the roughly 6 million Wikidata entities that have an entry in the English Wikipedia.

Next, we manually create templates for the 100 most frequent Wikidata predicates. For example, for the predicate P19 ("place of birth"), we create the template $S$ was born in $O$ and generate English statements by filling the $S$ and $O$ slots with entities

\footnotetext{
${ }^{2}$ As opposed to the LM acquiring world knowledge implicitly as a side effect of its training objective.

${ }^{3}$ As opposed to generating or retrieving a surface form which may or may not correspond to an entity.
}

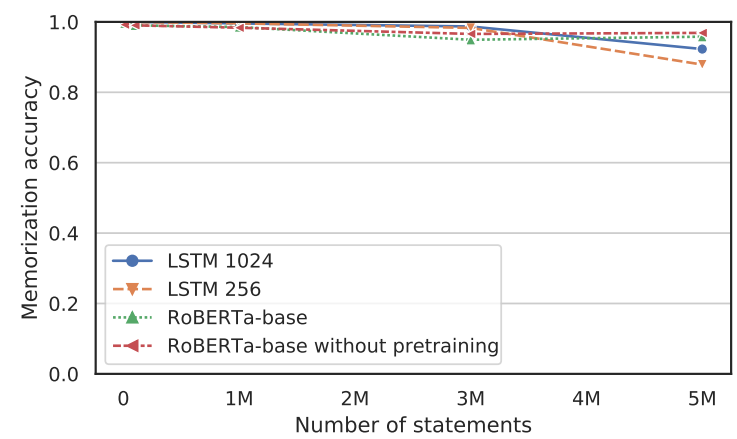

Figure 2: Accuracy of statement memorization with symbolic representation of 1 million entities.

from the sets defined above for which this relation holds. ${ }^{4}$ To make queries for an object unique given subject and predicate, we arbitrarily select exactly one fact if there are multiple objects and discard the other facts. This process yields 5 million statements involving up to 1 million entities, and 10 million statements involving up to 6 million entities. These statements then serve as training instances, i.e., given the query "Barack Obama was born in [MASK]", the model should predict Hawaii. As our goal is to store facts in a LM, there is no distinction between training and test data.

Models and training. We consider two common LM architectures: LSTMs (Hochreiter and Schmidhuber, 1997) and Transformers (Vaswani et al., 2017). For LSTMs, we compare two configurations; a randomly initialized two-layer LSTM with layers size 256 (LSTM 256) and one with layer size 1024 (LSTM 1024). For Transformers, we compare a pretrained RoBERTa-base (Liu et al., 2019), and RoBERTa without pretraining, i.e., a randomly initialized Transformer of the same size. For consistent tokenization across all four models, we subword-tokenize statements with the RoBERTa tokenizer. To store statements in a LM, we train until the model reaches 99 percent memorization accuracy, i.e., overfits the training data almost perfectly, or stop early if accuracy does not improve for 20 epochs. See Appx. D for training details.

\subsection{Symbolic Representation}

With symbolic representation, each entity is represented as an entry in the LM's vocabulary. Prediction is done via masked language modeling (Devlin et al., 2019), by encoding the query with the LM, projecting the final hidden state of the [MASK] token onto the vocabulary and then taking a softmax

\footnotetext{
${ }^{4}$ Templates and sample of statements in Appx. B and C.
} 
over the vocabulary. As the results show (Fig. 2), symbolic representation yields very high memorization accuracies with a vocabulary of 1 million entities. Randomly initialized RoBERTa-base without pretraining works best, memorizing 97 percent of 5 million statements correctly.

Unfortunately, the softmax computation becomes prohibitively slow as the vocabulary size increases (Morin and Bengio, 2005), making symbolic representation with a softmax over a vocabulary consisting of the full set of 6 million Wikipedia entities impractical. Imposing a hierarchy is a common approach for dealing with large vocabularies, but did not work well in this case (See Appx. F.1).

\subsection{Surface Form Representation}

With surface form representation, each entity is represented by its canonical name. ${ }^{5}$ Since this name generally consists of more than one token, we cast memorizing statements and querying facts as a sequence-to-sequence task (Sutskever et al., 2014): Given the source sequence "Bert is a character on [MASK]", the model should generate the target sequence "Sesame Street". 6 To make models memorize statements, we train until perplexity on the training data reaches 1.0 or does not improve for 20 epochs. For evaluation, we generate the target sequence - i.e., the answer to a given query via a beam search with beam size 10 . We measure perfect-match accuracy of the full entity name, i.e., there is no credit for partial token matches.

The four models under comparison are now treated as sequence-to-sequence encoders and extended with a decoder of the same size: LSTM decoders for LSTM encoders (LSTM2LSTM) and randomly initialized Transformers for Transformer encoders (RoBERTa2Transformer, Transformer2Transformer).

Unlike symbolic representation, surface representation can handle the entire set of 6 million Wikipedia entities. As with symbolic representation, the randomly initialized Transformer (Fig. 3, dash-dotted red line) has the highest capacity, memorizing 10 million statements with 90 percent accuracy. A pretrained encoder (RoBERTa2Transformer) appears to have a deleterious effect, yielding lower accuracies than the randomly initialized Transformer2Transformer. While the larger LSTM2LSTM (layer size 1024) almost

\footnotetext{
${ }^{5}$ We use English Wikidata labels as canonical names.

${ }^{6}$ The [MASK] token is included since the target entity does not always occur at the end of a statement.
}

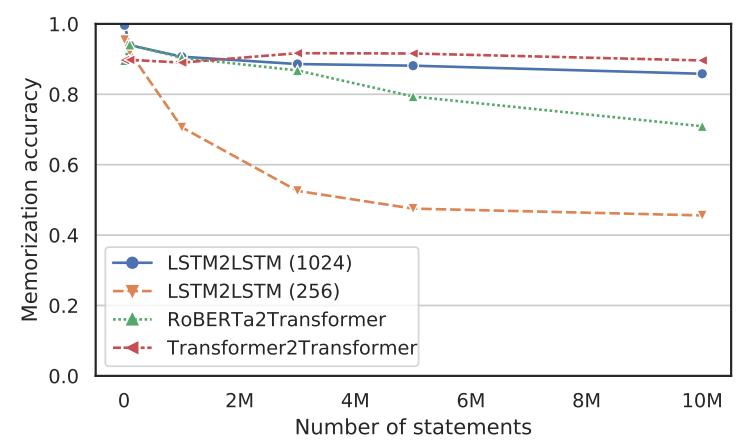

Figure 3: Accuracy of statement memorization with object entities represented by surface forms.

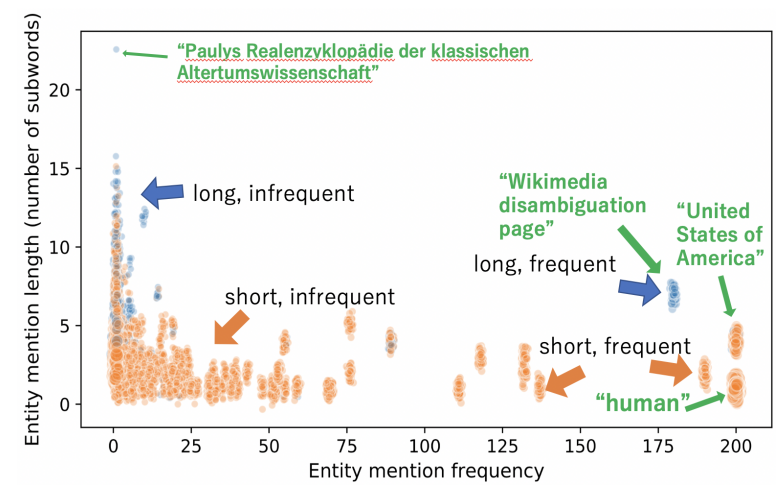

Figure 4: Error analysis of statements memorized via surface form representation. Correctly memorized statements orange, wrong ones blue. Selected clusters are annotated with the statement's object entity (green). Frequencies clipped to 200, jitter applied for clarity.

matches the performance of the best Transformer model, the smaller one (layer size 256) has insufficient capacity, memorizing less than 50 percent of 5 million statements.

Analysis of the Transformer2Transformer model (Fig. 4) reveals, perhaps unsurprisingly, that statements involving infrequent, long entity mentions are difficult to memorize. ${ }^{7}$ For example, the model fails to memorize most entity mentions that occur only in one to ten statements and have a length of 12 or more subwords (blue cluster, upper left).

\subsection{Continuous Representation}

With continuous representation, an entity $e_{i}, i \in$ $\left[1, N_{\text {entities }}\right]$ is represented by a $d$-dimensional embedding $\mathbf{y}_{i} \in \mathbb{R}^{d}$. After encoding a query with the LM, prediction is performed by projecting the final hidden state corresponding to the [MASK]

\footnotetext{
${ }^{7}$ We speculate that this drawback can be mitigated by shortening canonical names while ensuring a one-to-one mapping to entities, but leave this to future work.
} 


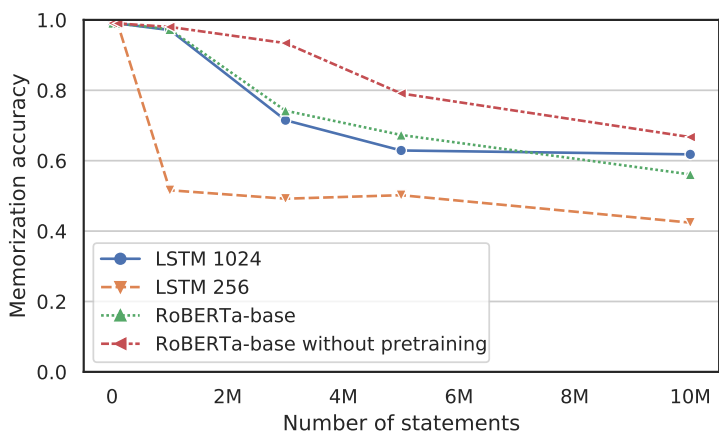

Figure 5: Accuracy of statement memorization with continuous entity representation.

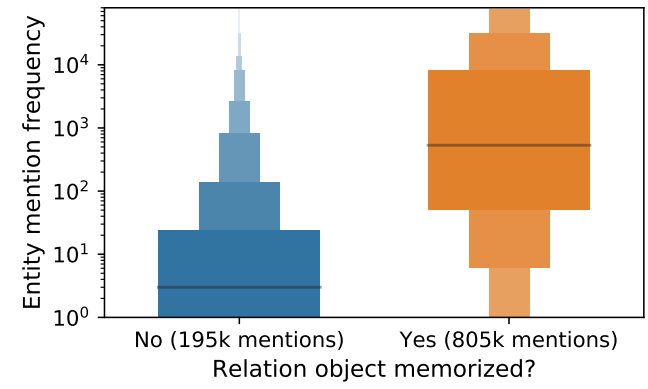

Figure 6: Error analysis of a sample of 1 million statements memorized by a randomly initialized Transformer with continuous representation.

token onto $\mathbb{R}^{d}$, obtaining the predicted embedding $\hat{\mathbf{y}} \in \mathbb{R}^{d}$. We use fixed, pretrained entity embeddings and train with cosine loss $L=1-\cos \left(\hat{\mathbf{y}}, \mathbf{y}_{\mathbf{i}}\right)$. At test time, the model prediction $\hat{\mathbf{y}}$ is mapped to the closest pretrained entity embedding $\mathbf{y}_{i}$ via nearest-neighbor search (Johnson et al., 2017).

Continuous prediction with fixed, pretrained embeddings. When training randomly initialized embeddings with a similarity objective, a degenerate solution is to make all embeddings the same, e.g., all-zero vectors. To prevent this, it is common practice to use negative samples (Bordes et al., 2013). When using fixed, pretrained embeddings as supervision signal, negative sampling is not necessary, since the target embeddings are not updated and therefore cannot become degenerate.

Wikidata embeddings. We train embeddings for 6 million Wikidata entities using feature-specific autoencoders to encode entity features such as names, aliases, description, entity types, and numeric attributes, following prior work on multi-modal KB embeddings (Pezeshkpour et al., 2018) and KB embeddings with autoencoders (Takahashi et al., 2018). Embedding training is detailed in Appx. E.
Results. Fig. 5 shows memorization accuracies achieved with continuous representation. Like surface representation, continuous representation scales to 6 million entities, and we see the same relative order of models, but with overall lower accuracies. RoBERTa without pretraining has the highest capacity for storing world knowledge statements, memorizing 67 percent of 10 million statements, while the small LSTM 256 model has the lowest capacity, memorizing 42 percent. Although far from fully understood, sequence-to-sequence architectures are relatively mature, with highly-optimized toolkits and hyperparameter settings publicly available (Ott et al., 2019). In contrast, prediction of continuous representations is still in an early stage of research (Kumar and Tsvetkov, 2019). We therefore see these results as lower bounds for LM capacity with continuous representations.

By design, memorization with continuous representations does not rely on entity names, and hence, in contrast to surface form representation, does not lead to difficulties in handling entities with long names. However, as with surface form representation, infrequent entities are more difficult to memorize than frequent ones. Most of the memorization errors (Fig. 6, blue, left) involve infrequent entities with a median frequency of 3 , while most of the correctly memorized statements (orange, right) involve entities that occur more than 100 times.

\section{LM Capacity for Storing Facts}

We now turn to the second question, how model capacity scales with model size (Fig. 7, top). With a 12-layer Transformer of layer size 96 or 192 (top subfigure, solid red and dashed green lines), memorization accuracy quickly drops as the number of facts to memorize increases. Larger models can memorize more facts, but accuracy drops rather quickly, e.g., to 65 percent of 3 million facts memorized with a layer size of 384 (dotted orange line).

Assuming a desired memorization accuracy of 80 percent, we record the maximum number of facts a model of a given size can memorize at this level (Fig. 7, bottom). For the model sizes considered here, storage capacity appears to scale linearly, with a model of layer size 384 (55M parameters) storing one million facts and a model of layer size 960 (160M parameters) storing 7 million facts.

Apart from the number of facts to store, we hypothesize that successful storage depends on two more factors: the number of entities and the en- 

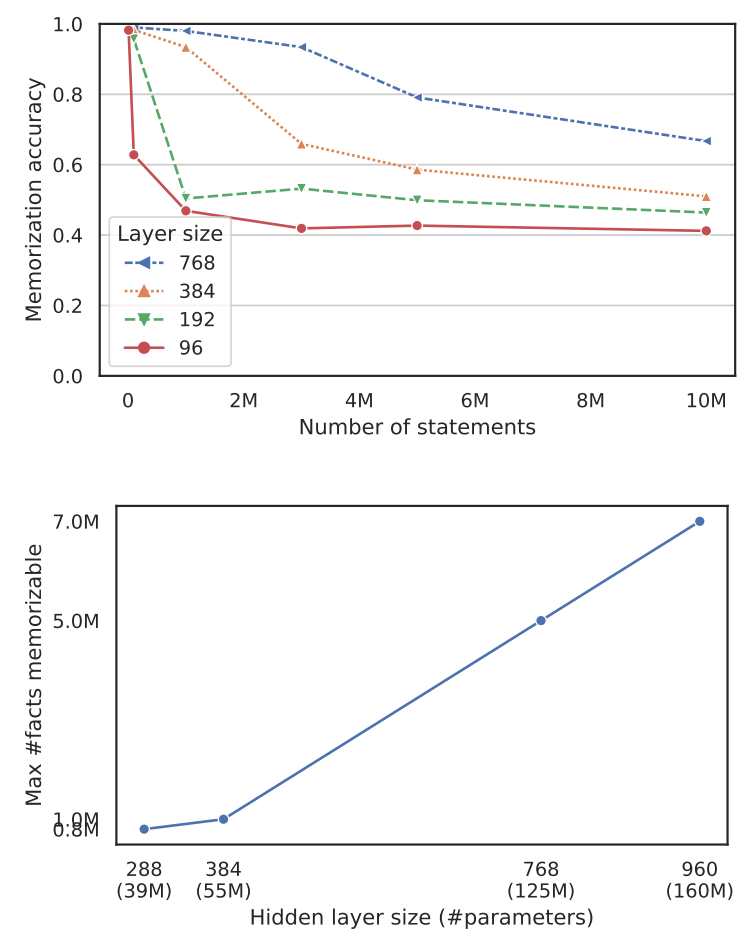

Figure 7: Scaling of storage capacity with model size. Memorization accuracy decreases as the number of facts grows (top). The maximum number of facts a model of a given layer size (parameter count) can memorize with an accuracy of 80 percent increases linearly (bottom). All models are 12-layer Transformer with continuous representation of 6 million entities.

\begin{tabular}{lrr}
\hline & \multicolumn{2}{c}{ Accuracy } \\
Representation & $1 \mathrm{M}$ & $6 \mathrm{M}$ \\
\hline Symbolic & 0.97 & $\mathrm{n} / \mathrm{a}$ \\
Surface & 0.92 & 0.90 \\
Continuous & 0.85 & 0.79 \\
\hline
\end{tabular}

Table 1: The number of entities (1M or $6 \mathrm{M}$ ) impacts memorization accuracy. The model is a 12-layer Transformer, layer size 768, memorizing 1 million facts.

tropy of their distribution. As expected, a large number of entities makes memorization more difficult (Table 1). The number of entities has a small effect with surface representation (2 percent drop), but with continuous representation accuracy drops from 85 percent to 79 percent when the number of entities increases from 1 to 6 million. We also observe an impact of the entity distribution (Appx. G), but leave detailed analysis to future work.

\subsection{Storage Efficiency}

Our comparison of different entity representations (§3) does not control for the number of trainable model parameters. That is, we selected common ar-

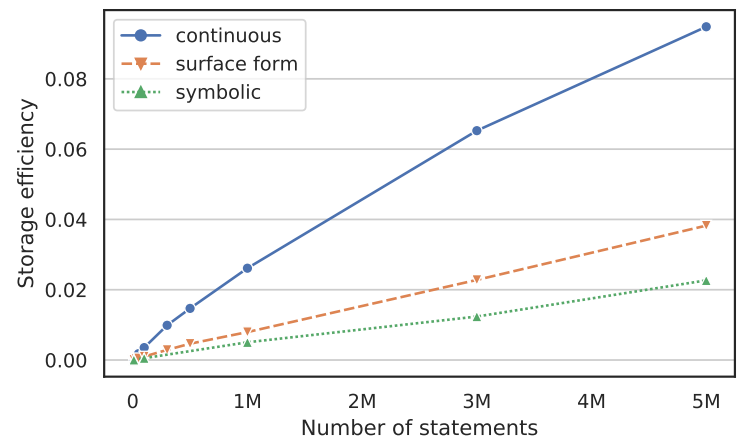

Figure 8: Storage efficiency with symbolic, surface form, and continuous representation of entities. In the setting considered in this work, continuous representation is most efficient.

chitectures, such as a Transformer with 12 layers of size 768 , but made no effort to ensure that, e.g., the number of trainable parameters introduced by the softmax layer in a model with symbolic representation matches the number of trainable parameters introduced by the addition of a sequence-to-sequence decoder component in a model with surface form representation. In order to more fairly compare entity representations across models with differing numbers of trainable parameters, we formulate the storage efficiency of a model designed to memorize statements:

$$
\text { Storage efficiency }=\frac{\# \text { statements } \times \text { accuracy }}{\# \text { parameters }}
$$

This measure expresses the intuition that a model is efficient if it requires few parameters to memorize a large number of statements with high accuracy. When quantifying efficiency with this measure, we find that continuous representation is the most efficient (Figure 8) and hence use this form of entity representation in the remainder of this work.

\section{Querying Stored Facts}

So far, we saw that it is possible to store millions of facts in a LM, by finetuning the model to predict the masked object of statements like Barack Obama was born in [MASK]. However, given the large number of model parameters and training effort, mere storage is not a compelling achievement: The underlying relations, here /Barack_Obama, wasBornln, Hawaii $\rangle$, can be stored more compactly and with perfect accuracy in a structured KB.

One of the potential benefits of the LM-as-KB paradigm is the LM's ability to handle paraphrases. If the LM's representation of the statement above is 
sufficiently similar to its representation of queries like Barack Obama is from [MASK] or Where is Barack Obama from? [MASK], this similarity could allow transfer from the memorized statement to these unseen queries. Is this soft querying of facts stored in a LM possible? We now conduct a controlled experiment to answer this question, expecting one of the following three outcomes:

1. Rote memorization. The model memorizes statements with little or no abstraction, so that even small, meaning-preserving changes to the query prevent the model from recalling the correct object.

2. Generic association. The model memorizes pairs of subject and object entities but disregards the predicate. For example, a model might predict Hawaii whenever the query contains the phrase Barack Obama, regardless of context. This pathological behaviour could be especially prevalent if the distribution of object entities co-occurring with a given subject is dominated by one object.

3. Fact memorization. The model memorizes facts expressed in statements by forming abstractions corresponding to entities and predicates. This allows retrieving a fact with a variety of queries.

Sections 3 and 4 already established that a model of sufficient size can perform rote memorization of millions of statements. We now design an experiment to test whether LMs are capable of fact memorization while taking care to distinguish this capability from generic association. Concretely, our goal is to test if a LM that has memorized a statement like Barack Obama was born in Hawaii can use this knowledge to answer a query like Barack Obama is from [MASK]. Conveniently, wasBornln relations are among the most frequent in Wikidata and hold for a diverse set of subject and object entities. This diversity of entities makes this predicate a good candidate for our case study, since statements involving a predicate with a less diverse set of subject or object entities are easier to memorize. ${ }^{8}$

Statements and controls. We sample 100k statements generated by the template " $\mathrm{S}$ was born in O". To allow distinguishing if a model that memorizes these 100k facts does so by generic assocation or by fact memorization, we introduce control facts. Given a fact $\langle\mathrm{S}, \mathrm{P}, \mathrm{O}\rangle$, its control $\left\langle\mathrm{S}, \mathrm{P}^{\prime}, \mathrm{O}^{\prime}\right\rangle$ involves the same subject $S$, but a distinct predicate $\mathrm{P}^{\prime}$ and object O'. For example, a control for

\footnotetext{
${ }^{8}$ For example, with the predicate is $A$ and relations like 〈Barack_Obama, isA, human〉 the model would do well by always predicting human if the subject mention matches a frequent person name pattern such as "two capitalized words".
}

the fact $\langle$ Albert_Einstein, wasBornln, Ulm $\rangle$ is the fact $\langle$ Albert_Einstein, diedln, Princeton〉. We add 100k control statements generated from the template "S died in O" and train RoBERTa-base to memorize all 200k statements with 98 percent accuracy. The combination of statements and controls counters generic association: To correctly answer the query "Albert Einstein died in [MASK]", the model needs to take into account the predicate, since two distinct objects are associated with Albert_Einstein.

Query variants. Next, we collect query variants, such as "S is from O" (row labels in Fig. 9, top). Expecting good transfer for variants that are similar to the original statement, we include variants with small changes, such as varying punctuation. As more diverse variants, we select frequent relation patterns, such as "S (b. 1970, O)", from the GoogleRE Corpus (Google, 2013), as well as a query in question form and queries with irrelevant or misleading distractors such as " $\mathrm{S}$ was born in $\mathrm{O}$, but died somewhere else". For each variant, we generate 100k queries by filling the $\mathrm{S}$ and $\mathrm{O}$ slots with the same entity pairs as the original statements. To balance statements and controls, we create control templates (row labels in Fig. 9, bottom) and generate a matching number of control statements. Transfer results. We evaluate knowledge transfer from memorized statements to query variants using RoBERTa-base (Fig. 9, top, left), measuring accuracy over the $100 \mathrm{k}$ statements generated with a target query variant template. To measure the effect of pretraining on transfer ability, we compare to RoBERTa-base without pretraining (Fig. 9, top, right). We consider zero-shot transfer without any finetuning towards the target query variant, and a finetuning setting, in which the LM is first trained to memorize all 100k original statements and then finetuned until it memorizes a small number of statements in the target query format. ${ }^{9}$

In the zero-shot setting (leftmost column), even small changes to the query lead to a drop in fact recall: Adding an ellipsis (4th row) causes the model to answer $95 \%$ of queries correctly, a $3 \%$ drop from 98\% memorization of the original statements (first row). Adding an exclamation mark (5th row) results in a $8 \%$ drop. For other paraphrases, e.g., $S$, who is from $O$ (7th row) and $S$ is from $O$, zero-shot transfer works only in $35 \%$ and $20 \%$ of cases, and

\footnotetext{
${ }^{9}$ Our experiments, which test whether a LM can transfer a memorized fact to given target paraphrases, are converse to the probing setup by Jiang et al. (2020), which aims to find the best paraphrase for querying a given fact from a LM.
} 



Figure 9: Transfer from memorized statements (top: "S was born in O", bottom: "S died in O") to query variants.

the question format (11th row) allows zero-shot transfer with $32 \%$ accuracy. For the remaining paraphrases, e.g., those with parentheticals or the distractor died, zero-shot transfer is poor, with accuracies ranging from $3 \%$ to $13 \%$.

A clear trend is visible: transfer works best for similar statements and worst for dissimilar ones. To quantify this trend, we compute a representation of a statement template by averaging over its $100 \mathrm{k}$ mean-pooled, LM-encoded statements, and then measure the Euclidean distance between the original template representation and the representation of a query variant template. Correlating Euclidean distance and accuracy of zero-shot transfer obtains a Pearson coefficient of -0.68 , indicating a strong negative correlation. That is, transfer tends to work well for paraphrased queries the LM deems similar to the originally memorized statement, but fails if the LM's representation of a query is too dissimilar to its representation of the original statement. This trend is also reflected in the finetuning setting, with less similar variants requiring up to 500 instances until the model achieves 90 percent accuracy (last row), while transfer to more similar variants works well after finetuning on 5 to 50 target instances.

When using RoBERTa without pretraining to memorize statements, knowledge transfer to query variants is much worse. While transfer still works for the most similar variants (right, top rows), less similar variants require more finetuning compared 
to pretrained RoBERTa (right, middle rows). Transfer does not work for the least similar variants, with accuracies as low as 1 to 4 percent even after finetuning with 500 instances (right, bottom rows). Similar results for control statements are shown in Fig. 9 (bottom). We take these results as evidence that pretraining enables LMs to handle paraphrased queries and that LMs can memorize facts beyond mere rote memorization and generic association.

\section{Limitations and Conclusions}

Limitations. This work is not without limitations. We only use one KB in our experiments. Arguably, as the largest publicly available source of world knowledge, Wikidata is the most promising resource for equipping LMs with such knowledge, but attempts to store a $\mathrm{KB}$ with different structure might result in different outcomes, since some types of graphs are easier to memorize for a LM than others (See Appx. G).

While we use language like "train a LM to memorize statements" for simplicity throughout this work, what we do in case of pretrained LMs is more akin to adaptive pretraining (Gururangan et al., 2020). It is possible that integrating entity supervision directly into LM pretraining (Févry et al., 2020) allows more efficient fact storage.

Our analysis was focused on entity representations and ignored the question of how to represent relation predicates or entire relation triples. Here, relation learning (Baldini Soares et al., 2019) and LM pretraining on fact-aligned corpora (Elsahar et al., 2018) are avenues for future work.

Finally, we formulated the LM-as-KB paradigm in terms of storing and retrieving relation triples. While structured KBs such as Wikidata consist of such triples and hence our experiments showing storage and retrieval of triples LMs are sufficient as a proof-of-concept in principle, structured KBs allow more complex queries than the ones considered here, such as 1-to-n relations, multihop inference, queries involving numerical ranges, or facts qualified by time and location (Hoffart et al., 2013).

Conclusions. We gave a positive answer to Petroni et al. (2019)'s question if language models can serve as knowledge bases. Arguing that treating LMs as KBs requires representing a large number of entities, storing a large number of facts, and the ability to query a fact with a variety of queries, we showed that current LM architectures fulfill these requirements when extended with a compo- nent for representing entities. In addition to the ability to handle paraphrased queries, we envision further benefits from the LM-as-KB paradigm. For example, the fact-memorization and paraphrasefinetuning setting introduced in Section 5 allows precise control over which facts a LM learns.

\section{Acknowledgments}

We thank the anonymous reviewers for helpful feedback. This work was supported by a Google Focused Research Award.

\section{References}

Sungjin Ahn, Heeyoul Choi, Tanel Pärnamaa, and Yoshua Bengio. 2016. A neural knowledge language model. CoRR, abs/1608.00318.

Livio Baldini Soares, Nicholas FitzGerald, Jeffrey Ling, and Tom Kwiatkowski. 2019. Matching the blanks: Distributional similarity for relation learning. In Proceedings of the 57th Annual Meeting of the Association for Computational Linguistics, pages 2895-2905, Florence, Italy. Association for Computational Linguistics.

Emily M. Bender and Alexander Koller. 2020. Climbing towards NLU: On meaning, form, and understanding in the age of data. In Proceedings of the 58th Annual Meeting of the Association for Computational Linguistics, pages 5185-5198, Online. Association for Computational Linguistics.

Yoshua Bengio, Réjean Ducharme, Pascal Vincent, and Christian Jauvin. 2003. A neural probabilistic language model. Journal of machine learning research, 3(Feb):1137-1155.

Jon Louis Bentley. 1975. Multidimensional binary search trees used for associative searching. Сотmunications of the ACM, 18(9):509-517.

Jonathan Berant, Andrew Chou, Roy Frostig, and Percy Liang. 2013. Semantic parsing on Freebase from question-answer pairs. In Proceedings of the 2013 Conference on Empirical Methods in Natural Language Processing, pages 1533-1544, Seattle, Washington, USA. Association for Computational Linguistics.

Antoine Bordes, Nicolas Usunier, Alberto GarciaDurán, Jason Weston, and Oksana Yakhnenko. 2013. Translating embeddings for modeling multirelational data. In Proceedings of the 26th International Conference on Neural Information Processing Systems - Volume 2, NIPS'13, page 2787-2795, Red Hook, NY, USA. Curran Associates Inc.

Tom B. Brown, Benjamin Mann, Nick Ryder, Melanie Subbiah, Jared Kaplan, Prafulla Dhariwal, Arvind Neelakantan, Pranav Shyam, Girish Sastry, Amanda 
Askell, Sandhini Agarwal, Ariel Herbert-Voss, Gretchen Krueger, Tom Henighan, Rewon Child, Aditya Ramesh, Daniel M. Ziegler, Jeffrey Wu, Clemens Winter, Christopher Hesse, Mark Chen, Eric Sigler, Mateusz Litwin, Scott Gray, Benjamin Chess, Jack Clark, Christopher Berner, Sam McCandlish, Alec Radford, Ilya Sutskever, and Dario Amodei. 2020. Language models are few-shot learners. CoRR, abs/2005.14165.

Danqi Chen, Adam Fisch, Jason Weston, and Antoine Bordes. 2017. Reading Wikipedia to answer opendomain questions. In Proceedings of the 55th Annual Meeting of the Association for Computational Linguistics (Volume 1: Long Papers), pages 1870 1879, Vancouver, Canada. Association for Computational Linguistics.

Sam Coppens, Miel Vander Sande, Ruben Verborgh, Erik Mannens, and Rik Van de Walle. 2013. Reasoning over SPARQL. In $L D O W$.

Leon Derczynski, Eric Nichols, Marieke van Erp, and Nut Limsopatham. 2017. Results of the WNUT2017 shared task on novel and emerging entity recognition. In Proceedings of the 3rd Workshop on Noisy User-generated Text, pages 140-147, Copenhagen, Denmark. Association for Computational Linguistics.

Tim Dettmers, Minervini Pasquale, Stenetorp Pontus, and Sebastian Riedel. 2018. Convolutional 2d knowledge graph embeddings. In Proceedings of the 32th AAAI Conference on Artificial Intelligence, pages 1811-1818.

Jacob Devlin, Ming-Wei Chang, Kenton Lee, and Kristina Toutanova. 2019. BERT: Pre-training of deep bidirectional transformers for language understanding. In Proceedings of the 2019 Conference of the North American Chapter of the Association for Computational Linguistics: Human Language Technologies, Volume 1 (Long and Short Papers), pages 4171-4186, Minneapolis, Minnesota. Association for Computational Linguistics.

Jeffrey L. Elman. 1990. Finding structure in time. Cognitive Science, 14(2):179-211.

Hady Elsahar, Pavlos Vougiouklis, Arslen Remaci, Christophe Gravier, Jonathon Hare, Frederique Laforest, and Elena Simperl. 2018. T-REx: A large scale alignment of natural language with knowledge base triples. In Proceedings of the Eleventh International Conference on Language Resources and Evaluation (LREC 2018), Miyazaki, Japan. European Language Resources Association (ELRA).

Thibault Févry, Livio Baldini Soares, Nicholas FitzGerald, Eunsol Choi, and Tom Kwiatkowski. 2020. Entities as experts: Sparse memory access with entity supervision. CoRR, abs/2004.07202.

Matthew Francis-Landau, Greg Durrett, and Dan Klein. 2016. Capturing semantic similarity for entity link- ing with convolutional neural networks. In Proceedings of the 2016 Conference of the North American Chapter of the Association for Computational Linguistics: Human Language Technologies, pages 1256-1261, San Diego, California. Association for Computational Linguistics.

Daniel Gillick, Sayali Kulkarni, Larry Lansing, Alessandro Presta, Jason Baldridge, Eugene Ie, and Diego Garcia-Olano. 2019. Learning dense representations for entity retrieval. In Proceedings of the 23rd Conference on Computational Natural Language Learning (CoNLL), pages 528-537, Hong Kong, China. Association for Computational Linguistics.

Google. 2013. Google relation extraction corpus. https://ai.googleblog.com/2013/04/ 50000 -lessons-on-how-to-read-relation. html. Accessed: 2020-10-07.

Suchin Gururangan, Ana Marasović, Swabha Swayamdipta, Kyle Lo, Iz Beltagy, Doug Downey, and Noah A. Smith. 2020. Don't stop pretraining: Adapt language models to domains and tasks. In Proceedings of the 58th Annual Meeting of the Association for Computational Linguistics, pages 8342-8360, Online. Association for Computational Linguistics.

Kelvin Guu, Kenton Lee, Zora Tung, Panupong Pasupat, and Ming-Wei Chang. 2020. Realm: Retrievalaugmented language model pre-training. CoRR, abs/2002.08909.

Gary G Hendrix, Earl D Sacerdoti, Daniel Sagalowicz, and Jonathan Slocum. 1978. Developing a natural language interface to complex data. ACM Transactions on Database Systems (TODS), 3(2):105-147.

Jonathan Herzig, Pawel Krzysztof Nowak, Thomas Müller, Francesco Piccinno, and Julian Eisenschlos. 2020. TaPas: Weakly supervised table parsing via pre-training. In Proceedings of the 58th Annual Meeting of the Association for Computational Linguistics, pages 4320-4333, Online. Association for Computational Linguistics.

Sepp Hochreiter and Jürgen Schmidhuber. 1997. Long short-term memory. Neural Comput., 9(8):1735-1780.

Johannes Hoffart, Yasemin Altun, and Gerhard Weikum. 2014. Discovering emerging entities with ambiguous names. In Proceedings of the 23rd international conference on World wide web, WWW 2014, Seoul, South Korea, pages 385-396.

Johannes Hoffart, Fabian M Suchanek, Klaus Berberich, and Gerhard Weikum. 2013. Yago2: A spatially and temporally enhanced knowledge base from wikipedia. Artificial Intelligence, 194:28-61.

Johannes Hoffart, Mohamed Amir Yosef, Ilaria Bordino, Hagen Fürstenau, Manfred Pinkal, Marc Spaniol, Bilyana Taneva, Stefan Thater, and Gerhard 
Weikum. 2011. Robust disambiguation of named entities in text. In Proceedings of the 2011 Conference on Empirical Methods in Natural Language Processing, pages 782-792, Edinburgh, Scotland, UK. Association for Computational Linguistics.

Jeremy Howard and Sebastian Ruder. 2018. Universal language model fine-tuning for text classification. In Proceedings of the 56th Annual Meeting of the Association for Computational Linguistics (Volume 1: Long Papers), pages 328-339, Melbourne, Australia. Association for Computational Linguistics.

Filip Ilievski, Piek Vossen, and Stefan Schlobach. 2018. Systematic study of long tail phenomena in entity linking. In Proceedings of the 27th International Conference on Computational Linguistics, pages 664-674, Santa Fe, New Mexico, USA. Association for Computational Linguistics.

Zhengbao Jiang, Frank F. Xu, Jun Araki, and Graham Neubig. 2020. How can we know what language models know? Transactions of the Association for Computational Linguistics, 8:423-438.

Jeff Johnson, Matthijs Douze, and Hervé Jégou. 2017. Billion-scale similarity search with gpus. CoRR, abs/1702.08734.

Jared Kaplan, Sam McCandlish, Tom Henighan, Tom B. Brown, Benjamin Chess, Rewon Child, Scott Gray, Alec Radford, Jeffrey Wu, and Dario Amodei. 2020. Scaling laws for neural language models. CoRR, abs/2001.08361.

Thomas N. Kipf and Max Welling. 2017. Semisupervised classification with graph convolutional networks. In 5th International Conference on Learning Representations, ICLR 2017, Toulon, France, April 24-26, 2017, Conference Track Proceedings. OpenReview.net.

Nikolaos Kolitsas, Octavian-Eugen Ganea, and Thomas Hofmann. 2018. End-to-end neural entity linking. In Proceedings of the 22nd Conference on Computational Natural Language Learning, pages 519-529, Brussels, Belgium. Association for Computational Linguistics.

Sachin Kumar and Yulia Tsvetkov. 2019. Von MisesFisher loss for training sequence to sequence models with continuous outputs. In Proc. of ICLR.

Yinhan Liu, Myle Ott, Naman Goyal, Jingfei Du, Mandar Joshi, Danqi Chen, Omer Levy, Mike Lewis, Luke Zettlemoyer, and Veselin Stoyanov. 2019. RoBERTa: A robustly optimized BERT pretraining approach. CoRR, abs/1907.11692.

Robert Logan, Nelson F. Liu, Matthew E. Peters, Matt Gardner, and Sameer Singh. 2019. Barack's wife Hillary: Using knowledge graphs for fact-aware language modeling. In Proceedings of the 57th Annual Meeting of the Association for Computational Linguistics, pages 5962-5971, Florence, Italy. Association for Computational Linguistics.
Federico López, Benjamin Heinzerling, and Michael Strube. 2019. Fine-grained entity typing in hyperbolic space. In Proceedings of the 4th Workshop on Representation Learning for NLP (RepL4NLP2019), pages 169-180, Florence, Italy. Association for Computational Linguistics.

Frederic Morin and Yoshua Bengio. 2005. Hierarchical probabilistic neural network language model. In Aistats, volume 5, pages 246-252.

Maximilian Nickel, Volker Tresp, and Hans-Peter Kriegel. 2011. A three-way model for collective learning on multi-relational data. In $I C M L$, pages 809-816. Omnipress.

Yusuke Oda, Philip Arthur, Graham Neubig, Koichiro Yoshino, and Satoshi Nakamura. 2017. Neural machine translation via binary code prediction. In Proceedings of the 55th Annual Meeting of the Association for Computational Linguistics (Volume 1: Long Papers), pages 850-860, Vancouver, Canada. Association for Computational Linguistics.

Myle Ott, Sergey Edunov, Alexei Baevski, Angela Fan, Sam Gross, Nathan Ng, David Grangier, and Michael Auli. 2019. fairseq: A fast, extensible toolkit for sequence modeling. In Proceedings of the 2019 Conference of the North American Chapter of the Association for Computational Linguistics (Demonstrations), pages 48-53, Minneapolis, Minnesota. Association for Computational Linguistics.

Bryan Perozzi, Rami Al-Rfou, and Steven Skiena. 2014. Deepwalk: Online learning of social representations. In Proceedings of the 20th ACM SIGKDD international conference on Knowledge discovery and data mining, pages 701-710.

Matthew Peters, Mark Neumann, Mohit Iyyer, Matt Gardner, Christopher Clark, Kenton Lee, and Luke Zettlemoyer. 2018. Deep contextualized word representations. In Proceedings of the 2018 Conference of the North American Chapter of the Association for Computational Linguistics: Human Language Technologies, Volume 1 (Long Papers), pages 2227-2237, New Orleans, Louisiana. Association for Computational Linguistics.

Matthew E. Peters, Mark Neumann, Robert Logan, Roy Schwartz, Vidur Joshi, Sameer Singh, and Noah A. Smith. 2019. Knowledge enhanced contextual word representations. In Proceedings of the 2019 Conference on Empirical Methods in Natural Language Processing and the 9th International Joint Conference on Natural Language Processing (EMNLPIJCNLP), pages 43-54, Hong Kong, China. Association for Computational Linguistics.

Fabio Petroni, Aleksandra Piktus, Angela Fan, Patrick Lewis, Majid Yazdani, Nicola De Cao, James Thorne, Yacine Jernite, Vassilis Plachouras, Tim Rocktäschel, and Sebastian Riedel. 2020. KILT: a benchmark for knowledge intensive language tasks. CoRR, abs/2009.02252. 
Fabio Petroni, Tim Rocktäschel, Sebastian Riedel, Patrick Lewis, Anton Bakhtin, Yuxiang Wu, and Alexander Miller. 2019. Language models as knowledge bases? In Proceedings of the 2019 Conference on Empirical Methods in Natural Language Processing and the 9th International Joint Conference on Natural Language Processing (EMNLPIJCNLP), pages 2463-2473, Hong Kong, China. Association for Computational Linguistics.

Pouya Pezeshkpour, Liyan Chen, and Sameer Singh. 2018. Embedding multimodal relational data for knowledge base completion. In Proceedings of the 2018 Conference on Empirical Methods in Natural Language Processing, pages 3208-3218, Brussels, Belgium. Association for Computational Linguistics.

Nina Poerner, Ulli Waltinger, and Hinrich Schütze. 2019. E-BERT: Efficient-yet-effective entity embeddings for BERT. CoRR, abs/1911.03681.

Alec Radford, Jeff Wu, Rewon Child, David Luan, Dario Amodei, and Ilya Sutskever. 2019. Language models are unsupervised multitask learners. Technical report, OpenAI.

Colin Raffel, Noam Shazeer, Adam Roberts, Katherine Lee, Sharan Narang, Michael Matena, Yanqi Zhou, Wei Li, and Peter J Liu. 2019. Exploring the limits of transfer learning with a unified text-to-text transformer. arXiv preprint arXiv:1910.10683.

Jonathan Raiman and Olivier Raiman. 2018. Deeptype: Multilingual entity linking by neural type system evolution. In Proceedings of the Thirty-Second AAAI Conference on Artificial Intelligence, (AAAI18), the 30th innovative Applications of Artificial Intelligence (IAAI-18), and the 8th AAAI Symposium on Educational Advances in Artificial Intelligence (EAAI-18), New Orleans, Louisiana, USA, February 2-7, 2018, pages 5406-5413. AAAI Press.

Sebastian Riedel, Limin Yao, Andrew McCallum, and Benjamin M. Marlin. 2013. Relation extraction with matrix factorization and universal schemas. In Proceedings of the 2013 Conference of the North American Chapter of the Association for Computational Linguistics: Human Language Technologies, pages 74-84, Atlanta, Georgia. Association for Computational Linguistics.

Adam Roberts, Colin Raffel, and Noam Shazeer. 2020. How much knowledge can you pack into the parameters of a language model? CoRR, abs/2002.08910.

Claude E. Shannon. 1948. A mathematical theory of communication. Bell Syst. Tech. J., 27(3):379-423.

Daniil Sorokin and Iryna Gurevych. 2018. Modeling semantics with gated graph neural networks for knowledge base question answering. In Proceedings of the 27th International Conference on Computational Linguistics, pages 3306-3317. Association for Computational Linguistics.
Ilya Sutskever, Oriol Vinyals, and Quoc V. Le. 2014. Sequence to sequence learning with neural networks. In Proceedings of the 27th International Conference on Neural Information Processing Systems - Volume 2, NIPS'14, page 3104-3112, Cambridge, MA, USA. MIT Press.

Ryo Takahashi, Ran Tian, and Kentaro Inui. 2018. Interpretable and compositional relation learning by joint training with an autoencoder. In Proceedings of the 56th Annual Meeting of the Association for Computational Linguistics (Volume 1: Long Papers), pages 2148-2159, Melbourne, Australia. Association for Computational Linguistics.

Théo Trouillon, Johannes Welbl, Sebastian Riedel, Éric Gaussier, and Guillaume Bouchard. 2016. Complex embeddings for simple link prediction. In Proceedings of the 33rd International Conference on International Conference on Machine Learning - Volume 48, page 2071-2080. JMLR.org.

Ashish Vaswani, Noam Shazeer, Niki Parmar, Jakob Uszkoreit, Llion Jones, Aidan N Gomez, Łukasz Kaiser, and Illia Polosukhin. 2017. Attention is all you need. In Advances in neural information processing systems, pages 5998-6008.

Pauli Virtanen, Ralf Gommers, Travis E. Oliphant, Matt Haberland, Tyler Reddy, David Cournapeau, Evgeni Burovski, Pearu Peterson, Warren Weckesser, Jonathan Bright, Stéfan J. van der Walt, Matthew Brett, Joshua Wilson, K. Jarrod Millman, Nikolay Mayorov, Andrew R. J. Nelson, Eric Jones, Robert Kern, Eric Larson, CJ Carey, İlhan Polat, Yu Feng, Eric W. Moore, Jake Vand erPlas, Denis Laxalde, Josef Perktold, Robert Cimrman, Ian Henriksen, E. A. Quintero, Charles R Harris, Anne M. Archibald, Antônio H. Ribeiro, Fabian Pedregosa, Paul van Mulbregt, and SciPy 1.0 Contributors. 2020. SciPy 1.0: Fundamental Algorithms for Scientific Computing in Python. Nature Methods, 17:261-272.

Denny Vrandečić and Markus Krötzsch. 2014. Wikidata: a free collaborative knowledgebase. Соттиnications of the ACM, 57(10):78-85.

Wenhan Xiong, Jingfei Du, William Yang Wang, and Veselin Stoyanov. 2019. Pretrained encyclopedia: Weakly supervised knowledge-pretrained language model. CoRR, abs/1912.09637.

Ikuya Yamada, Hiroyuki Shindo, Hideaki Takeda, and Yoshiyasu Takefuji. 2016. Joint learning of the embedding of words and entities for named entity disambiguation. In Proceedings of The 20th SIGNLL Conference on Computational Natural Language Learning, pages 250-259. Association for Computational Linguistics.

Zichao Yang, Phil Blunsom, Chris Dyer, and Wang Ling. 2017. Reference-aware language models. In Proceedings of the 2017 Conference on Empirical Methods in Natural Language Processing, pages 
1850-1859, Copenhagen, Denmark. Association for Computational Linguistics.

Zhengyan Zhang, Xu Han, Zhiyuan Liu, Xin Jiang, Maosong Sun, and Qun Liu. 2019. ERNIE: Enhanced language representation with informative entities. In Proceedings of the 57th Annual Meeting of the Association for Computational Linguistics, pages 1441-1451, Florence, Italy. Association for Computational Linguistics. 


\section{A Overview: world knowledge in natural language processing}

\begin{tabular}{|c|c|c|c|}
\hline Paradigm / Task & Input & Output & Models and objectives \\
\hline Language modeling & Text & Text & $\begin{array}{l}\text { Next word prediction (Shannon, 1948; Elman, 1990; Bengio } \\
\text { et al., 2003), masked token prediction (Devlin et al., 2019) }\end{array}$ \\
\hline LM-as-KB? & Text & $\begin{array}{l}\text { Text / single-token } \\
\text { entity name }\end{array}$ & Closed-book QA (LAMA probe, Petroni et al., 2019) \\
\hline Sequence-to-sequence & Text & Text & $\begin{array}{l}\text { Text-to-text transformer (T5, Raffel et al., 2019), closed-book } \\
\text { QA (Roberts et al., 2020) }\end{array}$ \\
\hline Retrieval & Text & Text, answer span & $\begin{array}{l}\text { Answer-span selection (Chen et al., 2017), retrieval-augmented } \\
\text { LM (Guu et al., 2020), open-book QA }\end{array}$ \\
\hline Entity replacement & $\begin{array}{l}\text { Text, entity men- } \\
\text { tion spans }\end{array}$ & Text & Detecting replaced entity mentions (Xiong et al., 2019) \\
\hline Entity linking (EL) & $\begin{array}{l}\text { Text, entity men- } \\
\text { tion spans }\end{array}$ & Target entity & $\begin{array}{l}\text { AIDA (Hoffart et al., 2011), neural EL (Francis-Landau et al., } \\
\text { 2016; Kolitsas et al., 2018) }\end{array}$ \\
\hline Entity embeddings & $\begin{array}{l}\text { Text, entity men- } \\
\text { tion spans }\end{array}$ & Entity embeddings & Joint embedding of entities and text (Yamada et al., 2016) \\
\hline $\begin{array}{l}\text { LM with entity embed- } \\
\text { dings }\end{array}$ & $\begin{array}{l}\text { Text, linked en- } \\
\text { tity mentions, en- } \\
\text { tity embeddings }\end{array}$ & Text & ERNIE (Zhang et al., 2019), E-BERT (Poerner et al., 2019) \\
\hline LM with integrated EL & $\begin{array}{l}\text { Text, entity embed- } \\
\text { dings }\end{array}$ & Text & KnowBert (Peters et al., 2019) \\
\hline LM-as-KB (this work) & $\begin{array}{l}\text { Natural language } \\
\text { query }\end{array}$ & Target entity & Fact memorization, paraphrased queries, closed-book QA \\
\hline Knowledge-aware LM & $\begin{array}{l}\text { Text, knowledge } \\
\text { (sub)graph }\end{array}$ & Target entity, text & $\begin{array}{l}\text { Neural Knowledge LM (Ahn et al., 2016), Reference-aware LM } \\
\text { (Yang et al., 2017), Knowledge graph LM (Logan et al., 2019) }\end{array}$ \\
\hline Semantic parsing & $\begin{array}{l}\text { natural language } \\
\text { query }\end{array}$ & $\begin{array}{l}\text { meaning represen- } \\
\text { tation, target entity }\end{array}$ & $\begin{array}{l}\text { SEMPRE (Berant et al., 2013), GNNs for KBQA (Sorokin and } \\
\text { Gurevych, 2018) }\end{array}$ \\
\hline Universal Schema & $\begin{array}{l}\text { relation triples, text } \\
\text { patterns }\end{array}$ & $\begin{array}{l}\text { entity tuple and re- } \\
\text { lation embeddings }\end{array}$ & Matrix factorization (Riedel et al., 2013) \\
\hline $\begin{array}{l}\text { Knowledge graph embed- } \\
\text { dings }\end{array}$ & relation triples & $\begin{array}{l}\text { node and edge em- } \\
\text { beddings }\end{array}$ & $\begin{array}{l}\text { Link prediction; RESCAL (Nickel et al., 2011), TransE (Bor- } \\
\text { des et al., 2013), ComplexE (Trouillon et al., 2016), ConvE } \\
\text { (Dettmers et al., 2018) }\end{array}$ \\
\hline Graph neural networks & $\begin{array}{l}\text { nodes, node fea- } \\
\text { tures, edges }\end{array}$ & node embeddings & $\begin{array}{l}\text { DeepWalk (Perozzi et al., 2014), graph neural networks (Kipf } \\
\text { and Welling, 2017) }\end{array}$ \\
\hline Knowledge graphs & nodes, edges & nodes, edges & $\begin{array}{l}\text { Storage and retrieval, SQL/SPARQL queries, symbolic reason- } \\
\text { ing (Coppens et al., 2013) }\end{array}$ \\
\hline
\end{tabular}

Table 2: Approaches for using world knowledge in natural language processing, ranging from unstructured, purely text-based approaches (top), over approaches that mix text and structured KBs to varying degrees (middle), to approaches operating on structured KBs (bottom). 


\section{B Templates for generating English statements from Wikidata relations}

\begin{tabular}{|c|c|c|c|}
\hline ID & Template & ID & Template \\
\hline P31 & $\mathrm{S}$ is an instance of $\mathrm{O}$ & $\mathrm{P} 413$ & $\mathrm{~S}$ plays the position $\mathrm{O}$ \\
\hline P106 & $\mathrm{S}$ has the occupation $\mathrm{O}$ & P26 & $\mathrm{S}$ is spouse of $\mathrm{O}$ \\
\hline P17 & $\mathrm{S}$ belongs to the country $\mathrm{O}$ & P1830 & $\mathrm{S}$ is owner of $\mathrm{O}$ \\
\hline $\mathrm{P} 131$ & $\mathrm{~S}$ is located in the administrative territorial entity $\mathrm{O}$ & P1454 & $\mathrm{S}$ has the legal form $\mathrm{O}$ \\
\hline $\mathrm{P} 27$ & $\mathrm{~S}$ is citizen of $\mathrm{O}$ & P206 & $\mathrm{S}$ is located in or next to body of water $\mathrm{O}$ \\
\hline P47 & $\mathrm{S}$ shares a border with $\mathrm{O}$ & P710 & $\mathrm{S}$ is a participant of $\mathrm{O}$ \\
\hline P19 & $\mathrm{S}$ was born in $\mathrm{O}$ & P1441 & $\mathrm{S}$ is present in the work $\mathrm{O}$ \\
\hline P161 & $\mathrm{S}$ has the cast member $\mathrm{O}$ & P1532 & $\mathrm{S}$ represents $\mathrm{O}$ when playing sport $\mathrm{O}$ \\
\hline P421 & $\mathrm{S}$ is located in time zone $\mathrm{O}$ & P86 & $\mathrm{S}$ was composed by $\mathrm{O}$ \\
\hline P166 & $S$ received the award $O$ & P840 & $\mathrm{S}$ is set in the location $\mathrm{O}$ \\
\hline P54 & $\mathrm{S}$ is a member of the sports team $\mathrm{O}$ & P172 & $\mathrm{S}$ belongs to the ethnic group $\mathrm{O}$ \\
\hline P20 & $\mathrm{S}$ died in $\mathrm{O}$ & P175 & $\mathrm{S}$ is performed by $\mathrm{O}$ \\
\hline $\mathrm{P} 136$ & $\mathrm{~S}$ has the genre $\mathrm{O}$ & P57 & $\mathrm{S}$ is directed by $\mathrm{O}$ \\
\hline P69 & $\mathrm{S}$ was educated at $\mathrm{O}$ & P1889 & $\mathrm{S}$ is different from $\mathrm{O}$ \\
\hline P1412 & $\mathrm{S}$ is a language spoken, written or signed in $\mathrm{O}$ & P162 & $\mathrm{S}$ is produced by $\mathrm{O}$ \\
\hline P190 & $\mathrm{S}$ is a twinned administrative body of $\mathrm{O}$ & P118 & $\mathrm{S}$ belongs to the league $\mathrm{O}$ \\
\hline P641 & $\mathrm{S}$ participates in the sport $\mathrm{O}$ & P58 & $\mathrm{S}$ is screenwritten by $\mathrm{O}$ \\
\hline $\mathrm{P} 150$ & $\mathrm{~S}$ contains the administrative territorial entity $\mathrm{O}$ & P551 & $\mathrm{S}$ has the residence $\mathrm{O}$ \\
\hline P463 & $\mathrm{S}$ is a member of $\mathrm{O}$ & P103 & $\mathrm{S}$ has the native language $\mathrm{O}$ \\
\hline P735 & $\mathrm{S}$ has the given name $\mathrm{O}$ & P2789 & $\mathrm{S}$ connects with $\mathrm{O}$ \\
\hline P1343 & $\mathrm{S}$ is described by source $\mathrm{O}$ & $\mathrm{P} 750$ & $\mathrm{~S}$ has the distributor $\mathrm{O}$ \\
\hline P361 & $\mathrm{S}$ is a part of $\mathrm{O}$ & P725 & $\mathrm{S}$ is voiced by $\mathrm{O}$ \\
\hline P159 & the headquarters of $\mathrm{S}$ are located in $\mathrm{O}$ & $\mathrm{P} 272$ & $\mathrm{~S}$ is produced by the company $\mathrm{O}$ \\
\hline P1344 & $\mathrm{S}$ is participant of $\mathrm{O}$ & P112 & $\mathrm{S}$ was founded by $\mathrm{O}$ \\
\hline P495 & $\mathrm{S}$ has the country of origin $\mathrm{O}$ & P452 & $\mathrm{S}$ belongs to the industrial sector $\mathrm{O}$ \\
\hline P39 & $\mathrm{S}$ held the position of $\mathrm{O}$ & P81 & $\mathrm{S}$ is connected to line $\mathrm{O}$ \\
\hline P910 & $\mathrm{S}$ has the main category $\mathrm{O}$ & P97 & $\mathrm{S}$ has noble title $\mathrm{O}$ \\
\hline P105 & $\mathrm{S}$ has the taxon rank $\mathrm{O}$ & P740 & $\mathrm{S}$ formed in the location $\mathrm{O}$ \\
\hline P527 & $\mathrm{S}$ has the part $\mathrm{O}$ & P360 & $\mathrm{S}$ is a list of $\mathrm{O}$ \\
\hline P108 & $\mathrm{S}$ is employed by $\mathrm{O}$ & P793 & $\mathrm{S}$ is associated with the significant event $\mathrm{O}$ \\
\hline P279 & $\mathrm{S}$ is a subclass of $\mathrm{O}$ & P915 & $\mathrm{S}$ was filmed at $\mathrm{O}$ \\
\hline P171 & $\mathrm{S}$ has the parent taxon $\mathrm{O}$ & P410 & $\mathrm{S}$ has military rank $\mathrm{O}$ \\
\hline P140 & $\mathrm{S}$ has the religion $\mathrm{O}$ & P1001 & $\mathrm{S}$ applies to the jurisdiction of $\mathrm{O}$ \\
\hline P407 & $\mathrm{S}$ is in the $\mathrm{O}$ language & P30 & $\mathrm{S}$ is located on the continent $\mathrm{O}$ \\
\hline P1303 & $\mathrm{S}$ plays the instrument $\mathrm{O}$ & P749 & $\mathrm{S}$ has parent organization $\mathrm{O}$ \\
\hline P1411 & $\mathrm{S}$ has been nominated for $\mathrm{O}$ & P1435 & $\mathrm{S}$ has heritage designation $\mathrm{O}$ \\
\hline P102 & $\mathrm{S}$ is a member of political party $\mathrm{O}$ & P53 & $\mathrm{S}$ belongs to the family of $\mathrm{O}$ \\
\hline P3373 & $\mathrm{S}$ is a sibling of $\mathrm{O}$ & P400 & $\mathrm{S}$ was developed for the platform $\mathrm{O}$ \\
\hline P1376 & $\mathrm{S}$ is the capital of $\mathrm{O}$ & P921 & $\mathrm{S}$ has the main subject $\mathrm{O}$ \\
\hline P509 & $\mathrm{S}$ died because of $\mathrm{O}$ & P37 & $\mathrm{S}$ has the official language $\mathrm{O}$ \\
\hline P937 & $\mathrm{S}$ works in $\mathrm{O}$ & P734 & $\mathrm{S}$ has the family name $\mathrm{O}$ \\
\hline P264 & $\mathrm{S}$ was produced by the record label $\mathrm{O}$ & & \\
\hline P119 & $\mathrm{S}$ is buried in $\mathrm{O}$ & & \\
\hline P138 & $\mathrm{S}$ is named after $\mathrm{O}$ & & \\
\hline P530 & $\mathrm{S}$ has diplomatic relations with $\mathrm{O}$ & & \\
\hline $\mathrm{P} 40$ & $\mathrm{~S}$ is a child of $\mathrm{O}$ & & \\
\hline P155 & $\mathrm{S}$ follows $\mathrm{O}$ & & \\
\hline P276 & $\mathrm{S}$ is located in $\mathrm{O}$ & & \\
\hline P156 & $\mathrm{S}$ is followed by $\mathrm{O}$ & & \\
\hline P36 & $\mathrm{S}$ has the capital $\mathrm{O}$ & & \\
\hline P1196 & $\mathrm{S}$ has the manner of death $\mathrm{O}$ & & \\
\hline P127 & $\mathrm{S}$ is owned by $\mathrm{O}$ & & \\
\hline P101 & $\mathrm{S}$ works in the field $\mathrm{O}$ & & \\
\hline P607 & $\mathrm{S}$ participated in the conflict $\mathrm{O}$ & & \\
\hline P364 & $\mathrm{S}$ is a film or TV show with the original language $\mathrm{O}$ & & \\
\hline P6379 & $\mathrm{S}$ has works in the collection $\mathrm{O}$ & & \\
\hline P1346 & $\mathrm{S}$ is a winner of the $\mathrm{O}$ & & \\
\hline $\mathrm{P} 22$ & $\mathrm{~S}$ is the father of $\mathrm{O}$ & & \\
\hline P137 & $\mathrm{S}$ is operated by $\mathrm{O}$ & & \\
\hline
\end{tabular}

Table 3: Templates used to generate English statements from Wikidata relations. 


\section{Random sample of English statements generated from Wikidata relations}

- The Underfall Yard is followed by English Electric Part One

- Gazi Beg is a child of Farrukh Yassar

- 2011 European Rowing Championships is followed by 2012 European Rowing Championships

- 2009 Yemeni tourist attacks is located in Shibam

- George Best - A Tribute is performed by Peter Corry

- Gamecock Media Group is owned by SouthPeak Games

- 2017-18 Sheffield Wednesday F.C. season is followed by 2018-19 Sheffield Wednesday F.C. season

- Nennslingen is located in or next to body of water Anlauter

- 2013-14 Xavier Musketeers men's basketball team is followed by 2014-15 Xavier Musketeers men's basketball team

- Shock to the System is a part of Cyberpunk

- 1918-19 Ohio Bobcats men's basketball team follows 1917-18 Ohio Bobcats men's basketball team

- Ramya Krishnan has the spouse Krishna Vamsi

- The Cloud Minders follows The Way to Eden

- Curve is followed by Somethingness

- Austin Road is named after John Gardiner Austin

- Dione juno has the parent taxon Dione

- Spirit Bound Flesh is followed by The Wake

- Sidnei da Silva has the given name Sidnei

- In Memoriam is performed by Living Sacrifice

- Tracks and Traces is followed by Live 1974

- Grumman Gulfstream I is operated by Phoenix Air

- Timeline of Quebec history has the part Timeline of Quebec history (1982-present)

- Edwin C. Johnson held the position of Lieutenant Governor of Colorado

- Here Comes the Summer follows Jimmy Jimmy

- In Custody is screenwritten by Anita Desai

- Bertie Charles Forbes is the father of Malcolm Forbes

- The Mambo Kings has the cast member Helena Carroll

- Carnival of Souls has the cast member Art Ellison

- 1995-96 Philadelphia Flyers season is followed by 1996-97 Philadelphia Flyers season

- John Harley is the father of Edward Harley, 5th Earl of Oxford and Earl Mortimer

- Jane Fellowes, Baroness Fellowes has the spouse Robert Fellowes, Baron Fellowes

- Francis of Assisi is buried in Basilica of San Francesco d'Assisi

- 1990 Maharashtra Legislative Assembly election follows 1985 Maharashtra Legislative Assembly election

- Makabana Airport is named after Makabana

- Calvin Booth was born in Reynoldsburg

- The Telltale Head is followed by Life on the Fast Lane

- Alajos Keserú is a sibling of Ferenc Keserú

- Long An contains the administrative territorial entity Châu Thành 


\section{Hyperparameter settings and replicability statement}

\begin{tabular}{|c|c|c|c|}
\hline Entity representation & Architecture & Hyper-param. & Value \\
\hline \multirow[t]{12}{*}{ Symbolic } & \multirow[t]{6}{*}{ LSTM } & layers & 2 \\
\hline & & hidden size & 256,1024 \\
\hline & & dropout & 0.0 \\
\hline & & learning rate & 0.001 \\
\hline & & lr-scheduler & plateau \\
\hline & & optimizer & Adam \\
\hline & \multirow[t]{6}{*}{ Transformer } & model name & RoBERTa-base \\
\hline & & layers & 12 \\
\hline & & hidden size & 768 \\
\hline & & learning rate & $5 e-5$ \\
\hline & & lr-scheduler & plateau \\
\hline & & optimizer & Adam \\
\hline \multirow[t]{17}{*}{ Surface form } & \multirow[t]{7}{*}{ LSTM } & layers (enc) & 2 \\
\hline & & hidden size (enc) & 256,1024 \\
\hline & & layers (dec) & 2 \\
\hline & & hidden size (dec) & 256,1024 \\
\hline & & learning rate & 0.001 \\
\hline & & lr-scheduler & plateau \\
\hline & & optimizer & Adam \\
\hline & \multirow[t]{10}{*}{ Transformer } & model name (enc) & RoBERTa-base \\
\hline & & layers $(\mathrm{enc})$ & 12 \\
\hline & & hidden size (enc) & 768 \\
\hline & & dropout & 0.0 \\
\hline & & model name (dec) & random init. \\
\hline & & layers $(\mathrm{dec})$ & 12 \\
\hline & & hidden size (dec) & 768 \\
\hline & & learning rate & $5 e-4$ \\
\hline & & lr-scheduler & inverse sqrt \\
\hline & & optimizer & Adam \\
\hline \multirow[t]{16}{*}{ Continuous } & \multirow[t]{8}{*}{ LSTM } & layers & 2 \\
\hline & & hidden size & 256,1024 \\
\hline & & dropout & 0.0 \\
\hline & & learning rate & 0.001 \\
\hline & & lr-scheduler & plateau \\
\hline & & optimizer & Adam \\
\hline & & entity emb. dim & 64 \\
\hline & & entity emb. trainable & no \\
\hline & \multirow[t]{8}{*}{ Transformer } & model name & RoBERTa-base \\
\hline & & layers & 12 \\
\hline & & hidden size & 768 \\
\hline & & learning rate & $5 e-5$ \\
\hline & & lr-scheduler & plateau \\
\hline & & optimizer & Adam \\
\hline & & entity emb. dim & 64 \\
\hline & & entity emb. trainable & no \\
\hline
\end{tabular}

Table 4: Hyperparameter settings used in our experiments. 


\section{E Embeddings of Wikidata entities}

\section{KB Features}

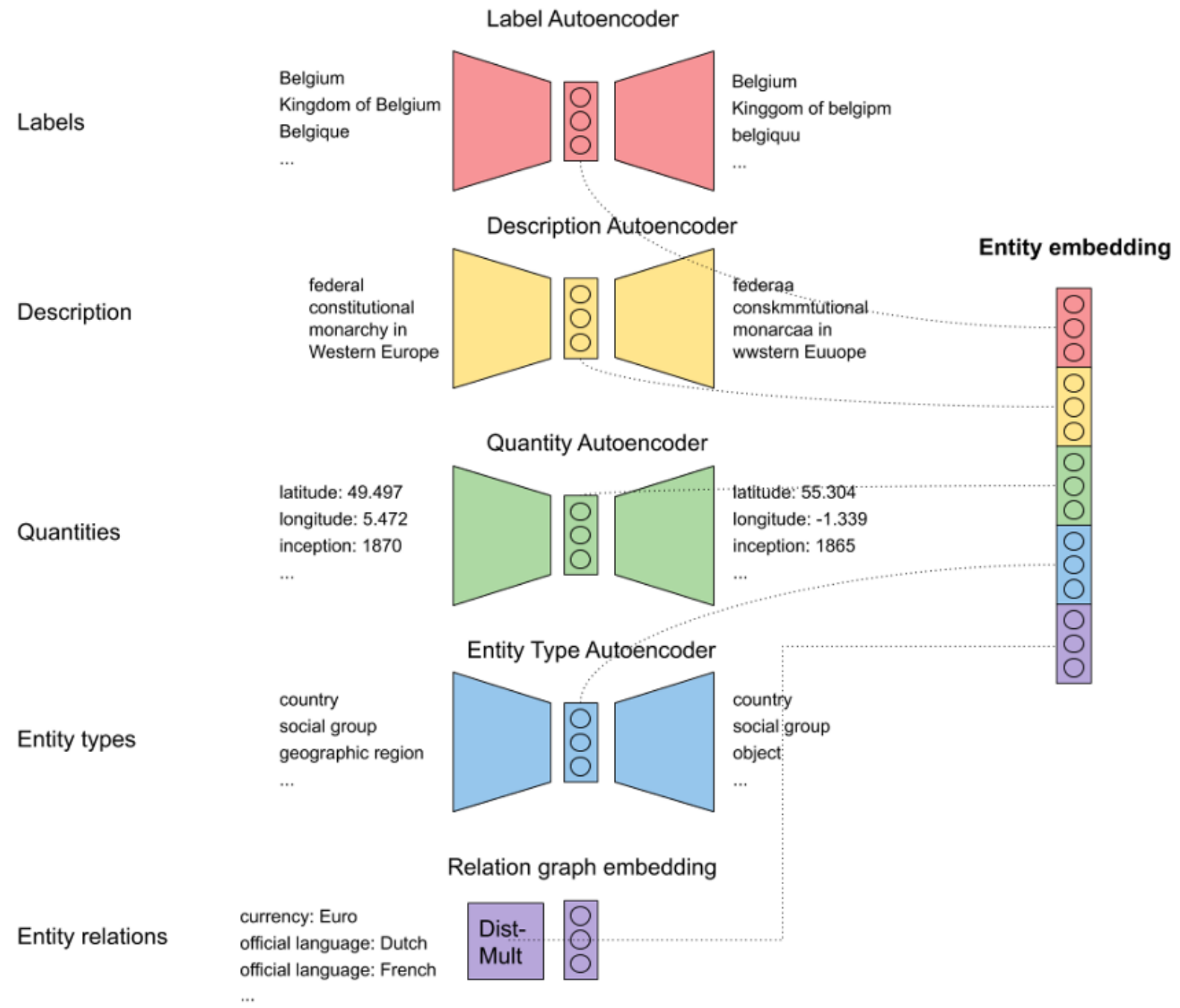

Figure 10: Training embeddings of Wikidata entities with feature-specific autoencoders.

We train the embedding of a given Wikidata entity by collecting its features from, encoding each feature to obtain a dense feature representation, and then concatenating feature representations. For textual features, we use RoBERTa-base as encoder and train corresponding decoders in a standard sequence-tosequence auto-encoding setup. For quantities, we select the 100 most common quantity types to obtain a fixed-sized representation and then follow a standard auto-encoding setup. Similarly we obtain a fixed-size entity type representation by selecting the 1000 most common entity types. The concatenated featurerepresentations are then compressed to embedding size $d$, using a separate autoencoder. Preliminary experiments with embedding sizes $d \in\{64,128,192,256\}$ showed similar memorization accuracies for all $d$, but faster convergence for smaller sizes. We set $d=64$ in our main experiments. 


\section{F Things that didn't work}

\section{F.1 Hierarchical entity representation with binary codes}

Since imposing a hierarchy is a common method for dealing with large vocabulary sizes (Morin and Bengio, 2005) in general, and large inventories of entities and entity types in particular (Raiman and Raiman, 2018; López et al., 2019), we created a hierarchy of all entities in Wikidata, using a given entity's position in this hierarchy as training signal. Specifically, we created the entity hierarchy by fitting a KD-tree (Bentley, 1975; Virtanen et al., 2020 ) with leaf size 1 over pretrained entity embeddings, thereby obtaining a binary partitioning of the embedding space in which each final partition contains exactly one entity embedding. The path from the KD-tree's root to a leaf can be represented as a binary code, which we use as training signal (Oda et al., 2017). Memorization accuracy of world knowledge facts with object entities represented in the form of these binary codes was substantially lower compared to the three approaches described in the main part of this work.

\section{F.2 Training entity embeddings with negative sampling}

Instead of using fixed, pretrained entity embeddings as training signal, we experimented with randomly initialized embeddings that are updated during training, using between 1 and 50 in-batch negative samples, which is a standard method in the knowledge base embedding literature (Bordes et al., 2013) and has been used successfully for entity retrieval (Gillick et al., 2019). However, compared to using fixed, pretrained entity embeddings without negative sampling, we observed lower memorization accuracies and slower convergence in our experiments.

\section{F.3 Updating pretrained entity embeddings during training}

Instead of using fixed entity embeddings, we tried updating them during training with in-batch negative sampling. This increased the number of trainable parameters, memory usage, and training time, but did not lead to higher memorization accuracies.

\section{F.4 Continuous representation with Euclidean distance loss}

Instead of normalizing entity embeddings to the unit hypersphere and training with cosine loss, we experimented with predicting the original pretrained entity embeddings and using the Euclidean distance as loss. Compared to using spherical entity embeddings as prediction targets, we observed slower convergence and lower memorization accuracies. 


\section{G Impact of graph type on memorizability}



Figure 11: Impact of graph type on a model's ability to memorize the graph. We consider two types of random graphs, namely a uniform (Erdos-Renyi) graph, and a scale-free (Barabasi) graph. We interpret graph edges as relation triples in a knowledge graph and train models to predict the relation object, given subject and predicate, until memorization accuracy reaches 99 percent. For a given number of model parameters, we gradually increase the number of relation triples to memorizes and record the maximum number of relation triples memorized for this number of parameters. We compare an LSTM, as well as a bilinear KB embedding (DistMult). For a given parameter budget, models are able to memorize more triples from a Erdos-Renyi graph (blue) than from a Barabasi graph, indicating that the latter is more difficult to memorize. 